Mitteilungen der Österreichischen Geographischen Gesellschaft, 158. Jg. (Jahresband), Wien 2016, S. 382-386

\title{
Verleihung der Franz-von-Hauer-Medaille an Axel BorSDorf
}

\author{
Helmut WoHLSCHLÄGL, Wien* \\ mit 1 Abb. im Text
}

Am 24. Juni 2016 fand im Festsaal der Universität Innsbruck im Rahmen einer feierlichen Veranstaltung zur Verabschiedung von Emer. O. Univ.-Prof. Dr. Axel BorsDorf (Institut für Geographie der Universität Innsbruck) die Verleihung der Franz-von-Hauer-Medaille statt. Die Medaille wurde vom Präsidenten der Österreichischen Geographischen Gesellschaft (ÖGG) persönlich im Anschluss an die nachfolgende Ansprache zur Würdigung des Geehrten überreicht.

Sehr geehrte Festgäste, werte Kolleginnen und Kollegen, sehr geehrte Damen und Herren!

Als Präsident der Österreichischen Geographischen Gesellschaft freue ich mich sehr, an dieser Festveranstaltung für Herrn Kollegen Axel BORSDORF teilnehmen und auch einen kleinen Beitrag

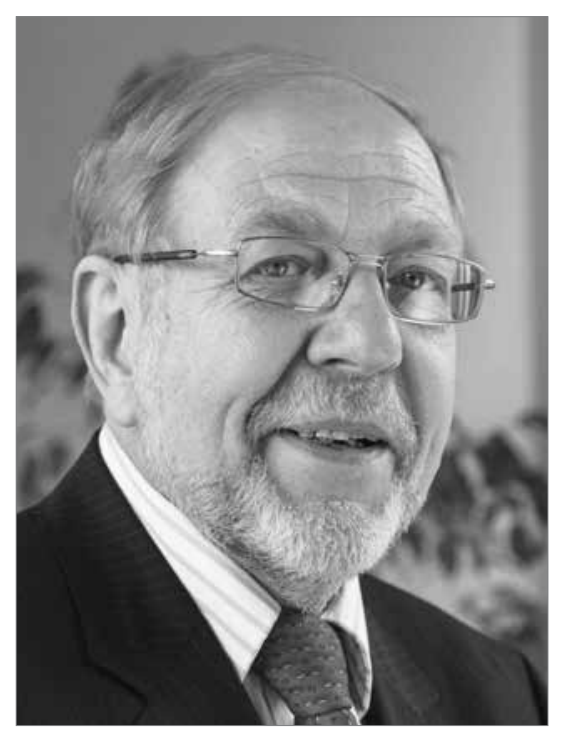

Axel BORSDORF

\footnotetext{
* Univ.-Prof. Mag. Dr. Helmut WoHLschläGL, Präsident der Österreichischen Geographischen Gesellschaft, Institut für Geographie und Regionalforschung der Universität Wien, Universitätsstraße 7/5, A-1010 Wien; E-Mail: helmut.wohlschlaegl@univie.ac.at, http://geographie.univie.ac.at
} 
zur seiner Würdigung leisten zu können. Im Programm der Festveranstaltung steht etwas kryptisch „Ehrung durch die Österreichische Geographische Gesellschaft“ und gar mancher mag sich vielleicht gefragt haben, um welche Ehrung es sich denn handelt. Nun kann das Geheimnis ja gelüftet werden - es handelt sich um die Verleihung der Franz-von-Hauer-Medaille, der höchsten und bedeutsamsten wissenschaftlichen Auszeichnung, die die Österreichische Geographische Gesellschaft $\mathrm{zu}$ vergeben hat.

Bisher ist die Franz-von-Hauer-Medaille in der insgesamt 160-jährigen Geschichte der ÖGG und der 122-jährigen der Medaille erst 48 mal verliehen worden. Sie deckt, gemäß § 3 der Statuten des Geographie-Fonds der ÖGG, den Bereich „geographisches Lebenswerk“ ab und ist dabei, wie es in den Statuten heißt, ,,neben der wissenschaftlichen Leistung auf die Würdigung institutioneller und organisatorischer Leistungen ausgerichtet“. Die Vergabe erfolgt auf der Basis von Nominierungen nach eingehender Prüfung durch die insgesamt elfköpfige Jury des Geographie-Fonds der ÖGG, die aus Vertreterinnen und Vertretern aller universitären Geographiestandorte in Österreich sowie aller Fachgruppen, Zweigvereine und Zweigstellen der ÖGG zusammengesetzt ist, in einem geheimen Abstimmungsverfahren.

Wieso Franz-von-Hauer-Medaille? Wer war Franz von HaUER? Dazu kurz ein paar Worte. Dr. Franz von HAuER war Mitglied der Akademie der Wissenschaften in Wien, Mitglied des Oberhauses (Herrenhauses) des österreichischen Reichsrats und von 1889 bis 1897 Präsident der 1856 gegründeten Vorgängergesellschaft der ÖGG, der „k.k. Geographischen Gesellschaft in Wien“. Er war ein sehr rühriger Präsident, trieb nicht nur die Förderung geographischer Forschung beträchtlich voran, sondern steigerte das Ansehen und die Bekanntheit der Geographie in der Öffentlichkeit ganz wesentlich, erhöhte dadurch die Mitgliederzahl der Gesellschaft, die damals eine Blütezeit erlebte, enorm und veranstaltete auch 1891 den 9. Deutschen Geographentag in Wien. (Anmerkung: Es dauerte dann 84 Jahre, bis wieder ein Geographentag in Österreich stattfand, und das war der 40. Geographentag, übrigens hier in Innsbruck 1975; im Jahr 2009 folgte dann der 57. Deutsche Geographentag in Wien.)

Anlässlich des 70. Geburtstags ihres rührigen Präsidenten im Jahr 1892 stiftete die k.k. Geographische Gesellschaft dann die Franz-von-Hauer-Medaille als ihre höchste wissenschaftliche Auszeichnung. Die Medaille wurde 1894 erstmals vergeben, und zwar an Erzherzog Franz Ferdinand für seine Weltumseglung und das von dieser mitgebrachte umfangreiche und wertvolle wissenschaftliche Material.

In den damaligen Statuten der Hauer-Medaille stand laut Stiftungsbeschluss: „Im Übrigen hat die Medaille die Bestimmung, von der k.k. Geographischen Gesellschaft an solche Personen (gleichviel ob In- oder Ausländer) verliehen zu werden, welche sich um die Erweiterung und Förderung des geographischen Wissens besonders verdient gemacht haben.“- und diesem Stiftungszweck folgt die ÖGG bis heute.

Wenn Kollege BORSDORF nun in wenigen Augenblicken die Hauer-Medaille verliehen erhält, dann zählt er zu einem sehr illustren Kreis der bisherigen Träger der Medaille, wie zum Beispiel, um nur einige zu nennen, Friedrich SiмоNy (der Inhaber der ersten Lehrkanzel für Geographie an einer österreichischen Universität, der Universität Wien, von 1851-1885, und Gründungsmitglied der ÖGG, damals k.k. Geographische Gesellschaft in Wien); wie zum Beispiel aber auch die Polarforscher Fridtjof Nansen, Julius PAYer, Roald Amundsen oder Alfred Wegener, oder die Wissenschafter Ferdinand von Richthofen, Fritz Machatschek, Otto Schlüter, Carl Troll, Hans Bobek und - in jüngerer Zeit - Elisabeth Lichtenberger (als bisher einzige Frau), Horst Mensching, Jürgen Bähr, Bruno Messerli, Peter Meusburger, Karl RupPert und zuletzt Martin Seger.

Aber - und das wollen wir an dieser Stelle natürlich nicht vergessen - auch aus dem Bereich der Innsbrucker Geographie gab es schon mehrere Träger der Franz-von-Hauer-Medaille, nämlich Franz von WIESER (1906, der erste Ordinarius für Geographie in Innsbruck an der Universität), Hans 
KINZL (1978, Begründer der „Innsbrucker Schule der historischen Bevölkerungsgeographie“ und auch Gebirgsforscher), sein Nachfolger auf dieser Professur, Adolf LeIDLMaIR (1989), der auch den 40. Deutschen Geographentag 1975 in Innsbruck veranstaltete, und nun wieder, als sein Nachfolger auf der gleichen Professur, Axel BorSDORF. Und noch ein weiterer Tiroler und bekannter Physiogeograph und Gebirgsforscher ist zu erwähnen, nämlich Helmut HeUBERGER, dem die Medaille im Jahr 2006 während der 150-Jahr-Feier der ÖGG verliehen wurde.

Die Nominierung von Univ.-Prof. Dr. Axel Borsdorf für die Vergabe der Franz-von-Hauer-Medaille erfolgte durch ein internationales Proponentenkomitee, das aus 14 führenden Wissenschaftern der Geographie aus Österreich, Deutschland und der Schweiz bestand. Als Begründung führte das Komitee an: „Aufgrund seiner herausragenden internationalen Verdienste um die Geographie als Wissenschaft, um die Weiterentwicklung des Faches Geographie an den österreichischen Universitäten (sowie an Universitäten im Ausland), um die geographischen Gesellschaften in Österreich, um die interdisziplinäre Lateinamerikaforschung sowie die internationale Gebirgsforschung beantragt das Proponentenkomitee die Verleihung der Franz-von-Hauer-Medaille an Herrn BorsDorf.“

Diese Verleihung erfolgte übrigens bereits in der Jurysitzung am 21. November 2014, im Jahr der Emeritierung von Prof. BorSDORF. Auf Wunsch der Innsbrucker Geographie wurde sie aber von der ÖGG bis heute geheim gehalten, um die Medaille erst im Rahmen dieser heutigen Festveranstaltung überreichen zu können.

Es würde an dieser Stelle zu weit führen, die wissenschaftlichen und wissenschaftsorganisatorischen Leistungen von Prof. BORSDORF ausführlich zu würdigen, auf ein paar Blitzlichter erlaube ich mir aber kurz hinzuweisen.

Nach seinem Studium in Göttingen und der Habilitation an der Universität Tübingen wurde Axel BorsDorf im Jahr 1991 als Nachfolger von Adolf LeIDLMAIR auf den Lehrstuhl für Geographie an der Universität Innsbruck berufen. Geprägt durch seinen akademischen Lehrer Herbert WILHELMY hatte BORSDORF bereits in Tübingen seinen regionalen Forschungsschwerpunkt in Südamerika, vor allem in Chile, gefunden sowie seine thematische Ausrichtung in der Siedlungs-, vor allem der Stadtgeographie, aber auch in methodischen Bereichen begründet. Innerhalb weniger Jahre gelang es ihm, ich zitiere jetzt aus dem Brief des Nominierungskomitees, ,wichtige und bleibende Akzente für eine konzeptionell-methodische Modernisierung und inhaltliche Neufokussierung der Innsbrucker Geographie zu setzen“.

Schon Mitte der 1990er Jahre wurde BORSDORF zunächst zum korrespondierenden und rasch danach zum wirklichen Mitglied der philosophisch-historischen Klasse der Österreichischen Akademie der Wissenschaften berufen. Sieben Jahre lang (1999 bis 2006) hatte er als Direktor - am Anfang zusätzlich zu seinen Lehrverpflichtungen in Innsbruck, später dann von der Universität Innsbruck für diese Funktion karenziert - die Leitung des Wiener Instituts für Stadt- und Regionalforschung der Österreichischen Akademie der Wissenschaften - in der Nachfolge von Elisabeth LICHTENBERGER - inne.

Während seiner Wiener Zeit hat BoRsDORF von 2004 bis 2006 auch die Präsidentschaft der Österreichischen Geographischen Gesellschaft - in der Nachfolge von Ingrid KRETSCHMER - übernommen. In seine Amtszeit fiel u.a. das Jubiläum zum 150-jährigen Bestehen der ÖGG. Mit der in Kooperation mit Frau KRETSCHMER durchgeführten Organisation zahlreicher Großveranstaltungen und mit der Förderung von Publikationen im Umfeld des Jubiläums setzte BorsDorf wichtige Akzente.

Zuvor, während der 1990er Jahre, war er bereits im Vorstand, teilweise auch als Vorsitzender, am Ausbau der Innsbrucker Geographischen Gesellschaft (IGG) - einem eigenständigen Zweigverein der ÖGG - maßgeblich beteiligt. Seit 2014 ist er auch einer der Ehrenvorsitzenden der IGG.

Überhaupt hat sich BORSDORF, und darauf möchte ich in meiner Funktion als Präsident einer geographischen Gesellschaft besonders hinweisen, während seines gesamten Wissenschaftslebens mit 
einer ausgesprochen umfangreichen Vortragstätigkeit für die geographischen Gesellschaften engagiert. Auch in Publikationen brachte er immer wieder zum Ausdruck, dass er in den geographischen Gesellschaften ein - vor allem im deutschsprachigen Raum - ganz wesentliches „Alleinstellungsmerkmal“ sieht, dessen „Vermittlungswirkung“ zwischen Wissenschaft, Öffentlichkeit, Politik und Praxis im Sinne der Einlösung des gesellschaftlichen Auftrags unseres Faches von größter Bedeutung ist. Seine grundsätzlichen Überlegungen zu diesem Thema hat er in einem sehr lesenswerten Aufsatz in der Festschrift zur 150-Jahr-Feier der ÖGG mit dem Titel „Geographische Gesellschaften in der Postmoderne" niedergelegt.

Ab 2006 übernahm BORSDORF die Leitung der neu gegründeten Forschungsstelle „Gebirgsforschung: Mensch und Umwelt“ der Österreichischen Akademie der Wissenschaften, die inzwischen unter seiner engagierten Leitung als „Institut für Interdisziplinäre Gebirgsforschung“ in Innsbruck etabliert ist und erheblich vergrößert wurde. Auf der Basis dieser immer zusätzlich zu seinen Lehrund Betreuungsaufgaben als Inhaber der Professur am Innsbrucker Universitätsinstitut für Geographie ausgefüllten Funktion fand BORSDORF zu einem zentralen Handlungsfeld in der eigenen Forschung und im Wissenschaftsmanagement: der interdisziplinären und internationalen Gebirgsforschung.

Aufbauend auf den eigenen Forschungen und den im Institut für Gebirgsforschung durchgeführten Studien und Analysen sowie gestützt auf ein weit gespanntes internationales Netzwerk ist es BORSDORF in den letzten Jahren ohne jeden Zweifel gelungen, die internationale Sichtbarkeit der österreichischen Gebirgsforschung erheblich zu steigern. Dadurch hat er einen wesentlichen Anteil daran, dass sich Innsbruck in den jüngsten Jahren - und jetzt zitiere ich wieder die Proponentengruppe - „zu einem wirklichen ,Leuchtturm“ einer modernen und auf wesentliche Zukunftsfragen ausgerichteten Gebirgsforschung entwickelt und weltweit positioniert hat". Nicht nur in diesem Zusammenhang ist für Axel BORSDORF aber auch der Wissenschaft-Praxis-Dialog mit einer umfangreichen Politikberatung immer von großer Bedeutung gewesen.

Zusätzlich - zum Teil aber auch im Zusammenhang mit der Gebirgsforschung - hat sich Axel BORSDORF auch mit großem Erfolg in der geographischen Lateinamerikaforschung engagiert, insbesondere zu stadtgeographischen Fragen oder zum Beispiel auch im Rahmen des von ihm (gemeinsam mit Christoph STADEL publizierten) jüngsten regionalgeographischen Werks, der regionalen Geographie des Andenraumes. BORSDORF hat zahlreiche Forschungsaufenthalte in Lateinamerika absolviert, immer wieder Gastprofessuren in Lateinamerika, vor allem in Chile, wahrgenommen und die Kooperation mit lateinamerikanischen - vor allem chilenischen - Universitäten (unter anderem in Universitätspartnerschaften dokumentiert) ausgebaut.

BORSDORF hat sich aber auch über viele Jahre hinweg im Vorstand des Österreichischen Lateinamerika-Instituts in Wien engagiert, hat am in Österreich einzigen Post-Graduate-Studium für Interdisziplinäre Lateinamerikastudien an der Universität Wien verantwortlich mitgewirkt und sich außerdem sehr für die Vernetzung und Selbstorganisation der interdisziplinären Lateinamerikaforschung in Österreich eingesetzt. Damit hat er einen ganz wesentlichen Anteil daran, dass in der Geographie, aber auch über die Geographie hinausgehend, der Standort Österreich einen gewichtigen Stellenwert in der internationalen und interdisziplinären Lateinamerikaforschung einnimmt.

Es würde den Rahmen sprengen, Axel BorSDORFs umfangreiche Tätigkeit als Mitglied wichtiger nationaler und internationaler Institutionen des Wissenschaftsmanagements, in Berufungskommissionen oder als Gutachter im In- und Ausland aufzulisten. Aber auch mit diesen Tätigkeiten, die ebenfalls wichtig sind, auch wenn sie nicht so plakativ erscheinen, hat BoRSDORF immer wieder Wesentliches für die österreichische Geographie leisten können.

Axel BorSDORF kann auf ein umfangreiches wissenschaftliches Werk verweisen - und dies sowohl in quantitativer Hinsicht als auch hinsichtlich der Breite der behandelten Themen und der Internationalität. Neben einer großen Zahl von Aufsätzen in bekannten Zeitschriften und in Sam- 
melbänden hat er mehrere Lehrbücher zu Teilgebieten der Geographie und zur Methodik (sogar zu Techniken des wissenschaftlichen Arbeitens in der Geographie in dem bereits in 2. Auflage erschienenen Buch „Geographisch denken und wissenschaftlich arbeiten“) verfasst. Auf die Tatsache, dass seine Lehr- und Betreuungstätigkeit sehr breit gestreut und sehr erfolgreich war, braucht man nicht eigens hinzuweisen. Sein besonderes Engagement galt mit zahlreichen Exkursionen und Geländepraktika der ,Schärfung ' geographischer Beobachtung und dem Verstehen geographisch-räumlicher, sozio-ökonomischer und sozialökologischer Zusammenhänge. Hinzuweisen ist auch darauf, dass sich Axel BorsDorf immer sehr für Fragen der Schulgeographie und des Schulfachs „Geographie und Wirtschaftskunde“ sowie der Fachdidaktik interessiert und diese Fachgebiete, soweit ihm dies möglich war, unterstützt und gefördert hat.

Vor diesem Hintergrund hielt es die Jury des Geographie-Fonds der ÖGG ohne Einschränkung für gerechtfertigt, dem Nominierungsvorschlag des bereits erwähnten Proponentenkomitees zu folgen, Herrn Emer. O. Univ.-Prof. Dr. Axel BORSDORF in Würdigung seines wissenschaftlichen Werkes, aber auch in Würdigung seiner herausragenden institutionellen und organisatorischen Leistungen im Sinne der Statuten des Geographie-Fonds der Österreichischen Geographischen Gesellschaft die Franz-von-Hauer-Medaille zu verleihen.

Ich freue mich nun, die Verleihungsurkunde der Österreichischen Geographischen Gesellschaft und die Franz-von-Hauer-Medaille überreichen zu können. Die Medaille besteht aus Silber und enthält auf der einen Seite ein eingraviertes Portrait von Franz von Hauer, auf der anderen Seite, umrahmt vom Schriftzug „Österreichische Geographische Gesellschaft" neben dem Datum des heutigen Tages die Gravur „Axel Borsdorf - Geograph. Gebirgs- Stadt- und Lateinamerikaforscher“, womit die zentralen Forschungsfelder des Geehrten auf der Medaille „,verewigt“ sind.

Ich gratuliere Kollegen BORSDORF sowohl namens der ÖGG als auch persönlich ganz herzlich zu seinen beeindruckenden fachwissenschaftlichen und wissenschaftsorganisatorischen Leistungen und wünsche ihm alles Gute auf seinem weiteren Lebensweg, gesundheitliches Wohlergehen und viel Erfolg bei seinen künftigen wissenschaftlichen Aktivitäten. 